\section{Eciture}

${ }^{1}$ Respiratory Department, Royal Devon and Exeter NHS Foundation Trust, Exeter, UK ${ }^{2}$ Department of Imaging, Hammersmith Hospital, Imperial College Healthcare NHS Trust, London, UK ${ }^{3}$ Department of Respiratory Medicine, North Devon District Hospital, Barnstaple, UK

\section{Correspondence to} Dr Charles Sharp, Respiratory Department, Royal Devon and Exeter NHS Foundation Trust, Barrack Road, Exeter EX2 5DW, UK;

Charles.sharp@doctors.org.uk

Accepted 24 May 2013

Published Online First

8 June 2013

To cite: Sharp C, Jackson J, Hands $\mathrm{G}$. Thorax

2014;69:399.

IMAGES IN THORAX

\title{
Congenital thoracic malformation
}

\author{
Charles Sharp, ${ }^{1}$ James Jackson, ${ }^{2}$ George Hands ${ }^{3}$
}

A 31-year-old man presented with recurrent chest pain. CT coronary arteriography (figure 1) demonstrated a well-defined area of hypertransradiancy at the left lung base supplied by a large artery arising from the thoracic aorta (figure 2) with no normal bronchial or pulmonary artery communication, but normal pulmonary venous drainage. The diagnosis is that of congenital bronchial atresia with a systemic artery supply (also termed intralobar sequestration). ${ }^{1}$

Congenital pulmonary abnormalities are being detected increasingly frequently as incidental findings during cross-sectional imaging performed for other reasons. Management is usually conservative unless complicated by infection or haemoptysis.

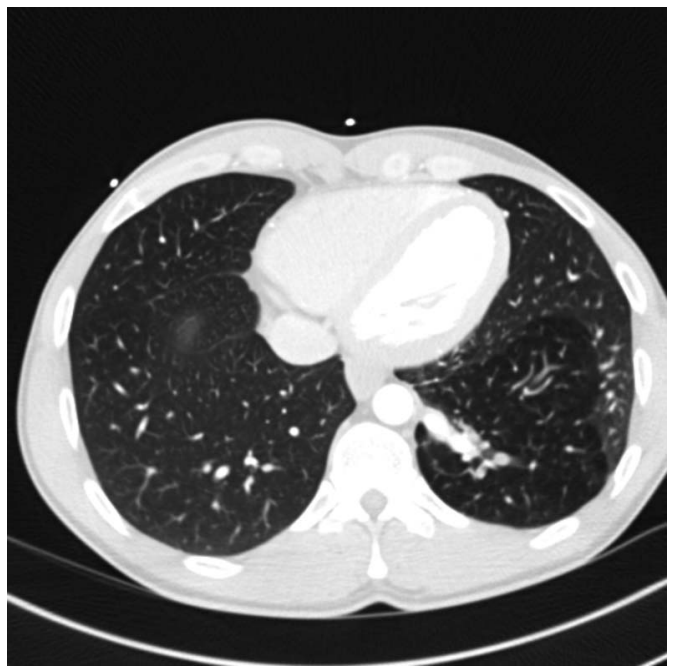

Figure 1 Axial CT section through the lung bases demonstrates a large well-defined area of hypertransradiancy at the left lung base.

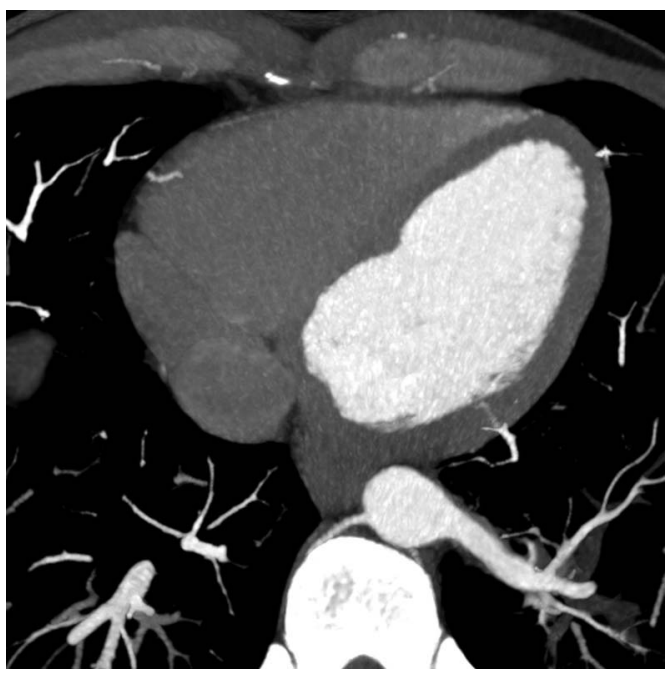

Figure 2 CT maximum intensity projection in the axial plane image during the aortic phase of contrast medium enhancement demonstrates the large supplying vessel arising from the descending thoracic aorta.

Contributors CS and GH conceived the article, JJ advised on image selection, CS wrote the text and all authors approved the final version. CS is guarantor of the content.

Competing interests None.

Patient consent Obtained.

Provenance and peer review Not commissioned; internally peer reviewed.

\section{REFERENCES}

1 Clements BS, Warner JO. Pulmonary sequestration and related congenital bronchopulmonary-vascular malformations: nomenclature and classification based on anatomical and embryological considerations. Thorax 1987;42:401-8. 